OPEN ACCESS

Edited by:

Fan Jin,

Zhejiang University, China

Reviewed by:

Alexandre Harlé,

Institut de Cancérologie de Lorraine,

France

Peng Ye,

Chengdu University, China

${ }^{*}$ Correspondence:

Christian Thiede

christian.thiede@uniklinikum-

dresden.de

Specialty section:

This article was submitted to

Genetics of Common and Rare

Diseases,

a section of the journal

Frontiers in Genetics

Received: 10 November 2021

Accepted: 13 December 2021

Published: 05 January 2022

Citation:

Stasik S, Mende M, Schuster C,

Mahler S, Aust D, Tannapfel A,

Reinacher-Schick A, Baretton G,

Krippendorf C, Bornhäuser $M$,

Ehninger G, Folprecht $G$ and Thiede $C$ (2022) Sensitive Quantification of Cell-

Free Tumor DNA for Early Detection of

Recurrence in Colorectal Cancer.

Front. Genet. 12:811291.

doi: 10.3389/fgene.2021.811291

\section{Sensitive Quantification of Cell-Free Tumor DNA for Early Detection of Recurrence in Colorectal Cancer}

\author{
Sebastian Stasik ${ }^{1,2}$, Marika Mende $^{1}$, Caroline Schuster ${ }^{3}$, Sandra Mahler ${ }^{1}$, Daniela Aust ${ }^{4}$, \\ Andrea Tannapfel ${ }^{5}$, Anke Reinacher-Schick ${ }^{6}$, Gustavo Baretton ${ }^{4}$, Claudia Krippendorf ${ }^{3}$, \\ Martin Bornhäuser ${ }^{1,2}$, Gerhard Ehninger ${ }^{1}$, Gunnar Folprecht ${ }^{1}$ and Christian Thiede ${ }^{1 *}$ \\ ${ }^{1}$ Medical Department I, University Hospital Carl Gustav Carus, Technische Universität, Dresden, Germany, ${ }^{2}$ National Center for \\ Tumor Diseases (NCT), Partner Site Dresden, Heidelberg, Germany, ${ }^{3}$ AgenDix GmbH, Dresden, Germany, ${ }^{4}$ Institute of \\ Pathology, University Hospital Carl Gustav Carus, Technische Universität, Dresden, Germany, ${ }^{5}$ Institute of Pathology, Ruhr \\ University, Bochum, Germany, ${ }^{6}$ Department of Hematology, Oncology and Palliative Care, St. Josef Hospital, Ruhr University, \\ Bochum, Germany
}

The detection of plasma cell-free tumor DNA (ctDNA) is prognostic in colorectal cancer (CRC) and has potential for early prediction of disease recurrence. In clinical routine, ctDNA-based diagnostics are limited by the low concentration of ctDNA and error rates of standard next-generation sequencing (NGS) approaches. We evaluated the potential to increase the stability and yield of plasma cell-free DNA (cfDNA) for routine diagnostic purposes using different blood collection tubes and various manual or automated cfDNA extraction protocols. Sensitivity for low-level ctDNA was measured in KRAS-mutant cfDNA using an error-reduced NGS procedure. To test the applicability of rapid evaluation of ctDNA persistence in clinical routine, we prospectively analyzed postoperative samples of 67 CRC (stage II) patients. ctDNA detection was linear between 0.0045 and $45 \%$, with high sensitivity (94\%) and specificity (100\%) for mutations at $0.1 \%$ VAF. The stability and yield of cfDNA were superior when using Streck BCT tubes and a protocol by Zymo Research. Sensitivity for ctDNA increased 1.5-fold by the integration of variant reads from triplicate PCRs and with PCR template concentration. In clinical samples, ctDNA persistence was found in $~ 9 \%$ of samples, drawn 2 weeks after surgery. Moreover, in a retrospective analysis of $14 \mathrm{CRC}$ patients with relapse during adjuvant therapy, we successfully detected ctDNA (median 0.38\% VAF; range $0.18-5.04 \%$ VAF) in $92.85 \%$ of patients significantly prior (median 112 days) to imaging-based surveillance. Using optimized preanalytical conditions, the detection of postoperative ctDNA is feasible with excellent sensitivity and allows the prediction of CRC recurrence in routine oncology testing.

Keywords: cell-free DNA, cell-free tumor DNA, liquid biopsy, next-generation sequencing, colorectal cancer, persistence and recurrence

\section{INTRODUCTION}

Cell-free DNA (cfDNA) represents a fraction of molecules that are constantly released into the blood circulation and other body fluids by cellular processes of necrosis or apoptosis (Schwarzenbach et al., 2011). In malignant diseases such as cancer, plasma cfDNA is enriched with circulating cell-free tumor DNA (ctDNA) and may carry tumor-derived genetic and epigenetic aberrations, reflecting the 
clonal architecture and evolution of corresponding cancer cells in the primary tumor tissue (Schwarzenbach et al., 2011; Crowley et al., 2013). Various molecular tumor-specific alterations have been detected in ctDNA, including DNA hypermethylation and point mutations in relevant genes such as KRAS and TP53 (Strickler et al., 2018; Luo et al., 2020).

As the release of ctDNA from tumor cells is related to the state and size of the tumor, levels of ctDNA may vary owing to cancer development and progression (Schwarzenbach et al., 2011; Crowley et al., 2013; Reinert et al., 2016). Consequently, the detection of ctDNA in plasma has potential to serve as a highly specific and low-invasive "liquid biopsy" for the early prediction of disease recurrence in clinical routine (Tie et al., 2016; Wan et al., 2017). The importance of ctDNA detection as prognostic biomarker has already been demonstrated in a variety of malignancies, including breast, lung, bladder, colon, and pancreatic cancer (Bettegowda et al., 2014). Due to its relevance as the second leading cause of cancer-related deaths and improvements in targeted therapies, plasma ctDNA has also been evaluated in colorectal cancer (CRC) with respect to risk stratification (Tie et al., 2016; Luo et al., 2020), prognosis (El Messaoudi et al., 2016; Wang et al., 2019), and prediction of treatment response or progression during postoperative surveillance (Siravegna et al., 2015; Tie et al., 2015; Reinert et al., 2016; Tie et al., 2016).

Although the presence of ctDNA is indicative for residual disease, the sensitive detection of ctDNA as early predictive marker is typically limited by the low frequency of mutant alleles in peripheral circulation (e.g., <0.1\%) and the low amount of available cfDNA as PCR template, respectively (Wan et al., 2017). Several next-generation sequencing (NGS) approaches potentially enable the quantification of mutant alleles at ultra-deep frequencies but often require high amounts of template cfDNA, complex molecular barcoding strategies, and/ or extensive bioinformatics, which impairs rapid translation into clinical practice (Kastrisiou et al., 2019). Furthermore, the quality and quantity of cfDNA as laboratory analyte is substantially affected by the short half-life time (typically $15 \mathrm{~min}-3 \mathrm{~h}$ ) and potential contamination with genomic DNA (gDNA) from white blood cells during the pre-analytical phase of blood collection (Medina Diaz et al., 2016; Wan et al., 2017).

In order to improve processing of plasma cfDNA for diagnostic purposes, we evaluated the potential to increase stability and yield of cfDNA for NGS downstream applications using adequate blood collection tubes (Streck, EDTA tubes) as well as various manual (Zymo Research, Qiagen, Analytik Jena) and automated (QIAsymphony) cfDNA extraction procedures. To come up with a rapid and robust method for routine laboratory testing, we performed a comprehensive evaluation of ctDNA detection (variable template concentrations and integration of variant reads from multiple PCR replicates) at variant allele frequencies in a clinically relevant range of 0.01-0.1\%, using an optimized error-reduced deep sequencing procedure (Stasik et al., 2018). To test the feasibility of our approach for early prediction of disease recurrence in clinical settings, 104 serial plasma samples of 14 patients with advanced CRC (stages II-IV) and relapse during adjuvant therapy post- tumor resection were retrospectively analyzed for the detection of known hot spot mutations (KRAS, NRAS, and TP53) in cfDNA and compared to imaging-based surveillance. In addition, we document the applicability of this approach for rapid prospective evaluation of ctDNA persistence in 67 patients diagnosed with stage II CRC.

\section{MATERIALS AND METHODS}

\subsection{Patient Samples}

All samples and clinical data were obtained with written informed consent of the patients. All studies involving human primary materials were performed after approval of the Local Ethical Board of the University Hospital Dresden and were in agreement with the Helsinki Declaration. For the analysis of postoperative ctDNA persistence (stage II CRC), patients were screened as part of the ColoPredict platform (AIO-KRK-0413) and subsequently registered for the ongoing prospective CIRCULATE-trial (NCT\#04089631).

\subsection{Blood Collection, Storage, and Plasma Preparation}

To optimize ctDNA preservation during blood collection, blood from a KRAS-mutant CRC patient, with a c.38G > A variant allele frequency (VAF) of 35\% (measured in peripheral blood by targeted sequencing), was diluted (1:1.75) in blood from healthy donors to obtain c.38G > A (p.Gly13Asp) VAFs of $\sim 20 \%$. Whole blood mixtures were aliquoted using different blood collection tubes: conventional S-Monovette EDTA tubes (Sarstedt, Germany) and Cell-Free DNA BCT tubes (Streck, Omaha, NE, United States) containing a special buffer for stabilization of nucleated blood cells. In order to monitor ctDNA stability, collection tubes ( 4 replicates $\mathrm{x} 7 \mathrm{ml}$ blood per tube) were subsequently incubated at room temperature $\left(22^{\circ} \mathrm{C}\right)$ for a period of up to 14 days and processed for cfDNA extraction at $0,24,48,72,96$, and $336 \mathrm{~h}$ post-collection. For separation of plasma, the blood samples were centrifuged at $300 \mathrm{~g}$ for $20 \mathrm{~min}$. Without disturbing the buffy coat, the plasma layer (supernatant) was carefully removed and transferred into a new 2-ml low-bind tube. To completely remove residual cells, plasma samples were re-centrifuged at $5,000 \mathrm{~g}$ for $10 \mathrm{~min}$, and the supernatant transferred to a new 2-ml low-bind tube and stored at $-20^{\circ} \mathrm{C}$ until cfDNA extraction.

\subsection{Extraction and Quantification of cfDNA}

To evaluate potential impacts of cfDNA extraction procedures on total extraction yield and the detection of mutant alleles, plasma samples of a KRAS c.38G > A (10\% VAF) positive CRC patient were processed using different manual protocols from various vendors: A Jena PME free-circulating DNA extraction kit (spinbased, carrier RNA: optional) (Analytik Jena, Jena, Germany), QIAamp Circulating NA Kit (vacuum-based, carrier RNA: yes) (Qiagen, Hilden, Germany), and Zymo Quick cfDNA serum and plasma kit (spin-based, carrier RNA: no) (Zymo Research, Irvine, CA, United States). In addition, cfDNA of matched plasma 
samples from CRC patients $(n=15)$ was extracted in parallel using the manual protocol by Zymo Research and an automated procedure on a QIAsymphony instrument (Qiagen) using the PAXcircDNA_STA_2400 protocol (Qiagen). All extractions were performed using $1-3 \mathrm{ml}$ of plasma according to the manufacturer's protocols. For all kits, cfDNA was eluted into $60 \mu \mathrm{L}$ ddH2O (or TE buffer), quantified by a $\beta$-globin-specific qPCR in comparison to a serial dilution of a reference DNA with a known quantity on a 7,500 Real-Time PCR System (Applied Biosystems, Foster City, CA, United States), and stored at $-20^{\circ} \mathrm{C}$ until downstream processing for NGS. All cfDNA concentrations are presented in $\mathrm{ng} \mathrm{mL}^{-1}$ plasma to adjust for different volumes of starting material.

\subsection{PCR Amplification and Deep Sequencing}

Sequencing of cfDNA samples was performed according to an optimized protocol for error-reduced NGS-based detection of low-level single-nucleotide variants (SNVs) on an Ion Torrent instrument, as described previously (Stasik et al., 2018). Briefly, Fusion PCR primers for the preparation of amplicon libraries were designed (Primer Premier 6; Premier Biosoft, Palo Alto, CA, United States) according to the manufacturer's recommendations (Fusion Method; Life Technologies). PCR on plasma cfDNA (40 cycles) was performed using the $\mathrm{Q}^{\circledR}$ High-Fidelity proofreading polymerases (New England Biolabs, Beverly, MA, United States). PCR primer sequences and specific PCR conditions for all target regions are listed in Supplementary Table S1. All PCRs were performed on a GeneAmp PCR System 9700 (Applied Biosystems, Foster City, CA, United States). After a two-round purification process with Agencourt AMPure XP Reagent (Beckman Coulter, Krefeld, Germany), barcoded PCR products were quantified with a Qubit 2.0 fluorometer (Life Technologies) using the Qubit dsDNA HS Assay (Life Technologies). For deep sequencing, $25 \mu \mathrm{L}$ of the diluted library $(30 \mathrm{pM})$ was loaded on an Ion Chef instrument (Life Technologies) for automatic template preparation and sequenced unidirectionally on an Ion S5 XL NGS system (Life Technologies), according to manufacturer's protocols and aiming at $\mathrm{a} \geq 100.000$-fold coverage per target region. Sequence data alignment of demultiplexed FastQ files, variant calling, and filtering was done using the Sequence Pilot software package (JSI Medical Systems GmbH, Ettenheim, Germany) with default settings. Human genome build HG19 (http://genome.ucsc.edu/) was used as reference genome for mapping algorithms. According to falsepositive rates of individual targets (Supplementary Table S2), NGS-based ctDNA detection was conducted with a defined cutoff of $0.01 \%$. VAFs below the predefined thresholds were considered ctDNA wild type (wt). Differences were analyzed using a two-sided Student t-test or the non-parametric Mann-Whitney $U$ test. A p value of $<0.05$ was considered significant. All calculations were conducted using Prism 5 (GraphPad, La Jolla, CA, United States) and SPSS Statistics 25 (IBM, Armonk, NY, United States).

\subsection{NGS Sensitivity for Low-Level ctDNA}

To quantify the linear range of NGS-based ctDNA detection, cfDNA from a KRAS c.35G > A mutant patient sample $(\sim 45 \%$
VAF) was serially diluted (10-fold) in wild-type (wt) cfDNA to obtain c.35G > A VAFs in the range of $0.0045-45 \%$. NGS sensitivity for low-level ctDNA was measured in KRAS c.35G $>$ A mutant cfDNA samples $(n=25)$ diluted to VAFs of $0.1 \%$. To address the impact of available cfDNA as PCR template for the detection of mutant alleles, 5 or 20 ng of cfDNA was used for PCR amplification. The control cfDNA $(n=9)$ from healthy individuals $(<50$ years of age $)$ served to assess per-base substitution error rates and specificity for the KRAS c.35G > A variant. To evaluate the potential benefit of integrating variant reads from multiple PCR replicates on NGS sensitivity, sequencing of 10 patient samples with the KRAS c.35G > A variant, diluted to $0.01 \% \mathrm{VAF}$, was performed using a pool of barcoded amplicons from triplicate PCR replicates ( $5 \mathrm{ng}$ template each reaction) for parallel sequencing. The detection of ctDNA was compared to the analysis of corresponding samples using a single PCR with $20 \mathrm{ng}$ of cfDNA as template.

\subsection{Clinical Validation of ctDNA-Based Detection of CRC Persistence and Recurrence}

In order to test the applicability of our approach for early detection of CRC recurrence in clinical settings, 104 serial plasma samples of patients $(n=14)$ with advanced CRC (stages II-IV) and relapse during adjuvant therapy were retrospectively analyzed for the detection of ctDNA. Time to detection of recurrence was compared between the detection of ctDNA and imaging-based surveillance. Furthermore, to evaluate the usability of ctDNA for rapid postoperative detection of CRC persistence, a prospective cohort of 67 patients with CRC stage II was screened for the detection of ctDNA in blood samples drawn $\sim 2$ weeks after complete resection of all evident tumor tissue. For all clinical samples, tumor-specific mutations were determined in a formalin-fixed paraffin-embedded (FFPE) tumor material by NGS panel analysis in a central pathological laboratory. For each patient, 1-3 tumor-informed mutations were selected for ctDNA monitoring. Processing of cfDNA was performed according to optimized conditions, which were initially determined in the methodical evaluations. Briefly, blood $(\sim 15 \mathrm{ml})$ was stored in Cell-Free DNA BCT (Streck) or PAXgene Blood ccfDNA tubes (Qiagen) and processed for plasma extraction within 7 days. cfDNA was extracted using the Zymo Quick cfDNA serum and plasma kit or the QIAsymphony instrument. PCR on individual samples was performed in triplicate using $\geq 5 \mathrm{ng}$ of cfDNA (40 cycles) and the Q5 proofreading polymerase. Pooled libraries were sequenced as described earlier.

\section{RESULTS}

\subsection{Optimizing Blood Collection for Stability of ctDNA}

The collection of blood in conventional EDTA tubes resulted in a 10- to 100-fold increase in extracted DNA (after 3 and 14 days), indicating an enhanced release of genomic DNA from the cellular fraction (Figure 1A). Consequently, the detection of mutant 

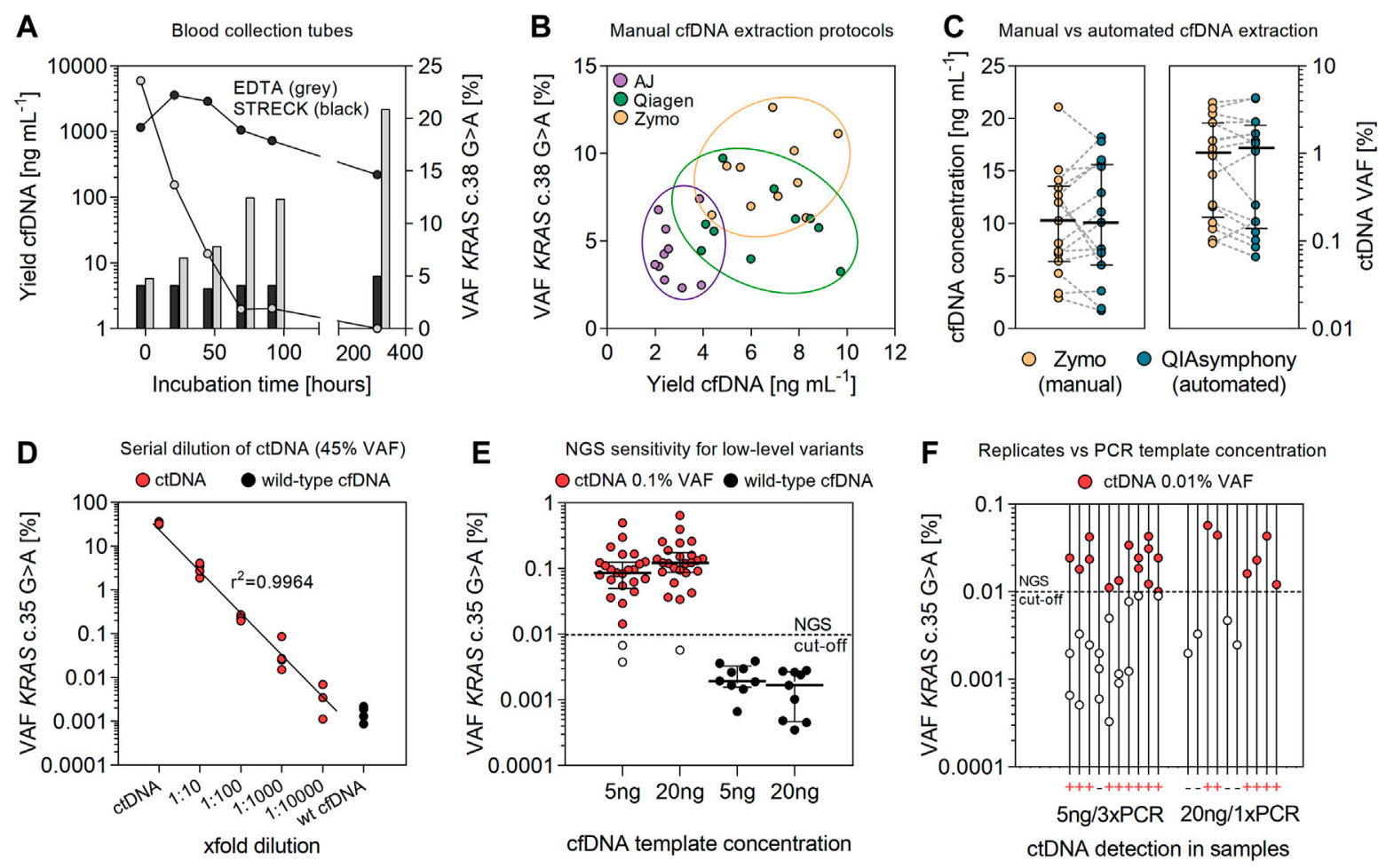

FIGURE 1 | Optimizing cfDNA preservation and extraction for sensitive detection of ctDNA. (A) Stability of ctDNA in EDTA and Streck blood collection tubes. Data for plasma levels of cfDNA [ng mL ${ }^{-1}$ plasma] and the frequency of corresponding $K R A S \mathrm{c} .38 \mathrm{G}>\mathrm{A}$ mutant alleles [\%] represent mean values of multiple replicates ( $n=4$ ). (B) Comparing the efficiency of manual cfDNA extraction kits for total extraction yield [ng $\mathrm{mL}^{-1}$ plasma] and the detection of ctDNA [\%]. Data are shown for different vendors: Analytik Jena (AJ), Qiagen, and Zymo Research. Extractions were performed in duplicate from plasma samples (1-3 ml) of CRC patients ( $n=5)$. (C) Extraction yield [ng mL ${ }^{-1}$ plasma] and concentrations of detected ctDNA [\%] in plasma samples $(2.5 \mathrm{ml})$ of CRC patients $(n=15)$ (median coverage 121,825; range 70,027-179,931 reads). Extraction of cfDNA was performed using the manual protocol by Zymo Research and on a QIAsymphony instrument. Error bars represent median values and interquartile range. (D) Serial dilution (10-fold) of ctDNA (45\% VAF) in wild-type cfDNA (median coverage 147,954; range 127,981-201438 reads). PCR was performed using the Q5 polymerase (NEB), 40 PCR cycles and 30 ng of cfDNA as PCR template. (E) NGS sensitivity for the detection of low-level ctDNA (0.1\% VAF) in cfDNA samples ( $n=25$ ) using 5 or 20 ng of cfDNA as template for PCR amplification (median coverage 114,830; range 71,321-323,158 reads). Wild-type cfDNA $(n=9)$ was used to measure the specific false-positive rate (substitution error) for the detection of the KRAS c.35G > A variant. (F) Detection of ctDNA at the NGS cutoff for quantification (0.01\% VAF) in cfDNA samples of CRC patients $(n=10)$ (median coverage 137,989; range 114,597-194,558 reads). Variant calling was performed using reads from a single PCR with $20 \mathrm{ng}$ cfDNA input or by the integration of sequencing reads from pooled triplicate PCRs and 5 ng of cfDNA as template.

alleles in EDTA collection tubes decreased significantly $(p<$ 0.0001 ) during blood incubation (half-life of $12-30 \mathrm{~h})$. Under these conditions, ctDNA was below detection limits $(<0.01 \%)$ after 14 days of incubation. In contrast, concentrations of extracted DNA (mean 4.08-4.56 $\mathrm{ng} \mathrm{ml}^{-1}$ plasma) and VAFs (mean 17.89-22.59\%) of the KRAS c.38G > A variant $(20 \%$ VAF) were stable for up to $96 \mathrm{~h}$ in Cell-Free DNA BCT (Streck) tubes, followed by a slight increase in DNA yield (mean $6.28 \mathrm{ng} \mathrm{ml}^{-1}$ ) and a concomitant decrease in KRAS VAFs (mean 14.64\%) measured at day 14.

\subsection{Comparison of Manual and Automated cfDNA Extraction Protocols}

The evaluation of manual cfDNA extraction protocols revealed significant differences in yield and stability of ctDNA (Figure 1B). Total yields of cfDNA from plasma samples (1-3 ml) were significantly higher $(p<0.0001)$ using procedures by Zymo Research (median 7.01; 95\% CI 5.69-8.03 $\mathrm{ng} \mathrm{ml}^{-1}$ ) and Qiagen (median 6.47; 95\% CI 4.98-8.05 $\mathrm{ng} \mathrm{ml}^{-1}$ ) than those using the protocol of Analytik Jena (median 2.42, 95\% CI 2.19-3.19 $\mathrm{ng} \mathrm{ml}^{-1}$ ). Similarly, the detection of mutant alleles with the KRAS c.38G > A mutation at $\sim 10 \%$ VAF was most accurate using the kit by Zymo Research (median 8.78; 95\% CI 7.33-10.30\% VAF) for cfDNA preparation, followed by Qiagen (median 5.86; 95\% CI 4.58-7.23\% $\mathrm{VAF}$ ) and AJ (median 3.96; 95\% CI 3.09-5.62\% VAF). No significant differences for cfDNA extraction yields (2.9-21.1 vs. $\left.1.7-18.2 \mathrm{ng} \mathrm{ml}^{-1} ; p=0.9807\right)$ and the detection of prevalent mutant alleles (0.09-3.84 vs. 0.06-4.35\% VAF; $p=0.9629)$ were observed for the preparation of matched plasma samples from CRC patients $(n=15)$ using the manual protocol by Zymo Research and an automated procedure on a QIAsymphony instrument (Figure 1C).

\subsection{NGS Sensitivity for the Detection of Low-Level ctDNA}

The detection of the KRAS c.35G > A variant in cfDNA was linear $\left(r^{2}=0.9964\right)$ between 0.0045 and $45 \%$ VAF (Figure 1D). The 
TABLE 1 | Retrospective validation of ctDNA-based detection of recurrence.

\begin{tabular}{|c|c|c|c|c|c|c|c|c|c|c|c|c|}
\hline \multicolumn{3}{|c|}{ Patient characteristic } & \multicolumn{5}{|c|}{ TNM classification } & \multicolumn{2}{|c|}{ ctDNA marker } & \multicolumn{3}{|c|}{$\begin{array}{l}\text { Time to detection of } \\
\text { recurrence }[\mathrm{d}]\end{array}$} \\
\hline Pat & Gender & Age (years) & UICC & pT & $\mathrm{pN}$ & $\mathbf{M}$ & Gene & HGVS & p.HGVS & Imaging & ctDNA & $\Delta$ \\
\hline Pat\#1 & Male & 77 & IV & 4 & 2 & 1 & KRAS & c. $35 G>A$ & p.Gly12Asp & 764 & 647 & 117 \\
\hline Pat\#2 & Male & 66 & IV & 4 & 2 & 1 & KRAS & c. $35 G>A$ & p.Gly12Asp & 962 & 962 & 0 \\
\hline Pat\#3 & Female & 81 & III & 3 & 1 & 0 & KRAS & c. $35 G>T$ & p.Gly12Val & 903 & 752 & 151 \\
\hline Pat\#4 & Female & 53 & IV & 3 & 0 & 1 & KRAS & c. $35 \mathrm{G}>\mathrm{T}$ & p.Gly12Val & 226 & 226 & 0 \\
\hline Pat\#5 & Female & 62 & $\|$ & 4 & 0 & 0 & KRAS & c. $34 \mathrm{G}>\mathrm{T}$ & p.Gly12Cys & 305 & n.d & n.a \\
\hline Pat\#6 & Female & 72 & IV & 3 & 1 & 1 & KRAS & c. $40 G>A$ & p.Val14lle & 163 & 109 & 54 \\
\hline Pat\#7 & Male & 74 & III & 3 & 1 & 0 & KRAS & C. $175 G>A$ & p.Ala59Thr & 268 & 42 & 226 \\
\hline Pat\#8 & Male & 63 & III & 3 & 1 & 0 & KRAS & c. $436 \mathrm{G}>\mathrm{A}$ & p.Ala146Thr & 228 & 116 & 112 \\
\hline Pat\#9 & Male & 70 & III & 3 & 2 & 0 & KRAS & c. $350 A>G$ & p.Lys117Arg & 397 & 315 & 82 \\
\hline Pat\#10 & Male & 54 & IV & 3 & 2 & 1 & NRAS & c. $38 \mathrm{G}>\mathrm{A}$ & p.Gly13Asp & 227 & 192 & 35 \\
\hline Pat\#11 & Male & 50 & IV & 4 & 1 & 1 & NRAS & c. $182 A>G$ & p.GIn61Arg & 463 & 326 & 137 \\
\hline Pat\#12 & Male & 54 & IV & 4 & 2 & 1 & TP53 & c.584T > C & p.lle195Thr & 427 & 318 & 109 \\
\hline Pat\#13 & Male & 77 & IV & 4 & 1 & 1 & TP53 & c. $743 G>A$ & p.Arg248GIn & 269 & 98 & 171 \\
\hline Pat\#14 & Male & 70 & IV & 3 & 1 & 1 & TP53 & c. $742 \mathrm{C}>\mathrm{T}$ & p.Arg248Trp & 381 & 195 & 186 \\
\hline
\end{tabular}

Abbreviations: UICC (Union for International Cancer Control); TNM (tumor (T), node (N), and metastasis (M)); HGVS (Human Genome Variation Society); $\triangle$ (difference between the detection of tumor progression between methods applied).

mean false-positive rate (substitution error) for the KRAS c.35G > A variant, measured in wild-type cfDNA, was $0.0016 \pm 0.0007 \%$ (Figure 1E; Supplementary Table S2). Applying the empirical rule for normal distribution, $99.73 \%$ of data observed will range within three standard deviations of the mean. According to that estimate, the specific limit for detection of the KRAS c.35G > A transition mutation in plasma cfDNA would correspond to a VAF of $0.0037 \%$ and one mutant allele out of 27,027 wild-type alleles, respectively. This is clearly below the predefined cutoff (0.01\% VAF) for SNV detection, previously determined for our error-reduced targeted NGS approach (Stasik et al., 2018). In line, KRAS mutant alleles (c.35G > A) at $0.1 \%$ VAF were detected with high sensitivity in 23/25 samples (94\%) in cfDNA of CRC patient samples. Specificity for the KRAS c.35G > A variant at $0.1 \% \mathrm{VAF}$ was 100\% (Figure 1E). Quantification of mutant alleles nonsignificantly ( $p=0.2000)$ increased using $20 \mathrm{ng}$ of cfDNA for amplification (median 0.12; 95\% CI 0.09-0.21\% VAF) compared to an input of $5 \mathrm{ng}$ cfDNA as a PCR template (median $0.08 ; 95 \%$ CI $0.06-0.15 \%$ VAF) (Figure 1E). The detection of low-level ctDNA in 10 samples with the KRAS c. $35 \mathrm{G}>\mathrm{A}$ variant at $\sim 0.01 \%$ VAF increased 1.5 -fold $(60-90 \%)$ by the integration of sequencing reads from pooled triplicate PCRs as compared to the analysis of a single PCR with higher amounts of cfDNA added per sample (Figure 1F).

\subsection{Retrospective Validation of ctDNA-Based Detection of Recurrence}

A total of 14 patients (10 males and 4 females) with advanced colorectal cancer (stages II-IV) and relapse during adjuvant therapy post-tumor resection were enrolled. The median age at tumor resection was 68 (range 50-81) years. Resected primary tumor samples were found positive for oncogenic hot spot mutations in KRAS $(n=9), \operatorname{NRAS}(n=2)$ and TP53 $(n=3)$. The median follow-up until imaging-based detection of recurrence was 381 (range 163-962) days. Demographic characteristics and pathological findings of patients are summarized in Table 1. In matched plasma cfDNA, tumorspecific point mutations were detected in $13 / 14$ patients $(92.85 \%)$ at the time of imaging-based detection of relapse (Table 1). No ctDNA was detected in one patient (Pat\#5) with a KRAS c.34G > T variant identified with $9 \% \mathrm{VAF}$ in the primary tumor. In general, levels of ctDNA increased with concentrations of total cfDNA, pointing to a correlation of cfDNA levels with tumor burden (Figure 2A). Significant differences were measured for levels of plasma cfDNA in patients without detectable ctDNA $<0.01 \%$, low-level ctDNA $0.01-0.1 \%(p=0.0061)$, and ctDNA $>0.1 \%\left(p=0.0003\right.$ ) (median cfDNA 6.07, 8.59, and $19.18 \mathrm{ng} \mathrm{ml}^{-1}$ plasma). For all patients monitored, ctDNA was below detection limits $(<0.01 \%)$ in samples $<1$ month post-tumor resection, demonstrating initial clearance of tumor-derived mutant alleles in blood circulation. Prior to clinical relapse, accumulation of ctDNA increased with daily rates of $0.003-0.012 \%$ (ctDNA d-1) (Figure 2B). The highest concentrations of plasma ctDNA were measured at the time of imaging-based detection of relapse, with a median VAF of $0.38 \%$ (range $0.18-5.04 \%$ ). The median delta between imaging-based and ctDNA-based detection of recurrence was 112 days $(p<0.0001$ ) (range 0-226 days) (Table 1; Figure 2C).

\subsection{Prospective Evaluation of ctDNA Persistence in Postoperative Samples}

For the prospective evaluation of postoperative ctDNA detection, 67 patients (CRC stage II, MSI low, median age of 69 years; range 27-85 years) were included. Postoperative blood sampling was conducted at a median of 13 (range 5-35) days after surgery (Figure 3A). Pathological findings of primary tumor material and results of the ctDNA analysis were available at a median of 30 (range 15-49) days and 40 (range 24-57) days after tumor resection, respectively. The most common molecular alterations used for ctDNA analysis were mutations in TP53 

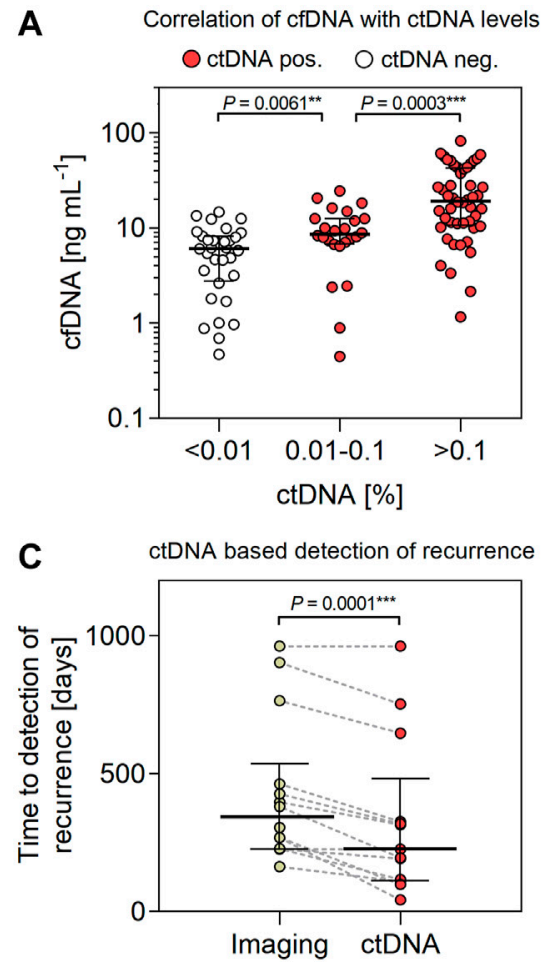
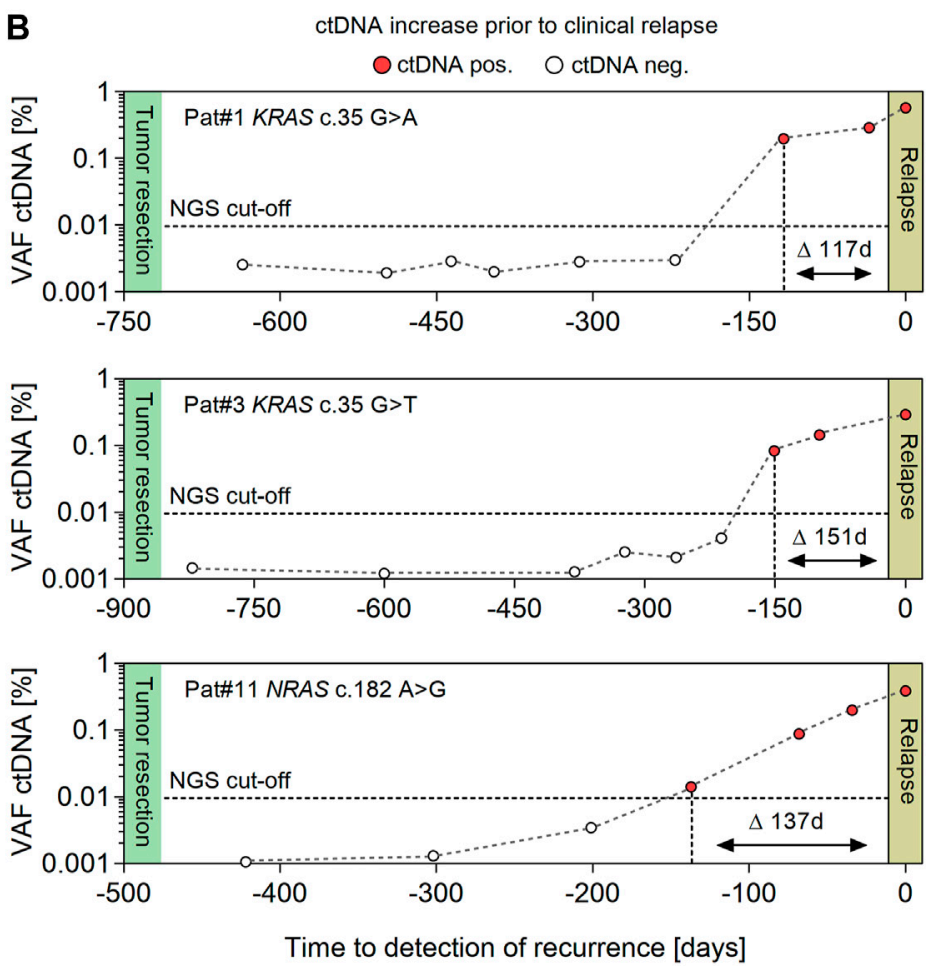

FIGURE 2 | Evaluating the feasibility of detecting ctDNA for early prediction of relapse in CRC patients. (A) Association of cfDNA with levels of ctDNA (<0.01\%, $0.01-0.1 \%$ and $>0.1 \%)$ in plasma samples $(n=104)$ of CRC patients $(n=14)$. (B) Exemplary illustration of ctDNA dynamics in $R A S-m u t a t e d$ CRC patients during adjuvant therapy post-tumor resection. The NGS detection limit is indicated at $0.01 \%$ VAF. (C) Comparing the progression free survival (PFS) of CRC patients $(n=14)$ assessed by conventional imaging-based diagnostics and NGS-based detection of ctDNA. Error bars represent median values and interquartile range.

(64\%), APC (42\%), and KRAS (30\%) (Figure 3B). For roughly half of patients $(48 \%)$, one single molecular marker was used for ctDNA screening, while two (or three in one case) eligible mutations were targeted in 52\% of patients. Out of the 67 patients, deep sequencing of cfDNA samples (median coverage of 486,240 , range $15,986-1,806,561$ reads per target) revealed the presence of postoperative ctDNA in 6 patients (9\%), with a median VAF of $0.042 \%$ (range $0.018-0.697 \%$ ) (Figure 3C; Supplementary Table S2). Generally, false-positive rates were below $0.01 \%$ VAF for all mutations analyzed (Figure 3C; Supplementary Table S2). However, substantial differences were measured for false-positive rates of individual mutations, which in part limits or qualifies their suitability as sensitive ctDNA markers. For example, background error was significantly lower for transversion mutations (median 0.0003, range $0-0.0024 \%$ ) and insertion/deletion variants (median 0 ) than for transition mutations (median 0.0016; range $0-0.0051 \%$ ) with the highest false-positive rate measured for the PIK3CA c.1633G > A variant (mean $0.0051 \pm 0.0012 \%$ StDiv) (Supplementary Figure S1; Supplementary Table S2).

\section{DISCUSSION}

In this study, we provide an easily adaptable, cost-effective, and rapid approach for the sensitive detection of plasma ctDNA in clinical routine, using adequate pre-analytical cfDNA processing and subsequent error-reduced deep sequencing. In addition, we document the translation of this approach for rapid prospective evaluation of ctDNA persistence in stage II CRC and the implementation of ctDNA for early prediction of disease recurrence.

The potential of ctDNA analysis as liquid biopsy in CRC for somatic tumor profiling (Strickler et al., 2018), the detection of treatment response (Siravegna et al., 2015; Tie et al., 2015; Reinert et al., 2016; Schøler et al., 2017; Tie et al., 2019), and disease progression (El Messaoudi et al., 2016; Tie et al., 2016; Reinert et al., 2019; Wang et al., 2019; Luo et al., 2020; Yeh et al., 2020) has been outlined previously. Typically, $15-20 \%$ of stage II CRC patients with curative resection will face disease recurrence (Böckelman et al., 2015), highlighting the need for sensitive monitoring strategies. We demonstrate an overall ctDNA persistence of $\sim 9 \%$ in stage II CRC patients after surgery, which confirms recent data on the frequency of postoperative ctDNA persistence (Tie et al., 2016; Reinert et al., 2019). In addition, tumor-informed ctDNA was detected 1-8 months prior to imaging-based detection of progression in $92.85 \%$ of CRC patients with relapse, which is in the range or superior to rates (72-92.3\%) determined previously (Tie et al., 2015; Schøler et al., 2017; Reinert et al., 2019; Wang et al., 2019; Luo et al., 2020; Yeh et al., 2020). Interestingly, in two patients (Pat. 2 and 4), no difference was observed between ctDNA- and imaging-based 


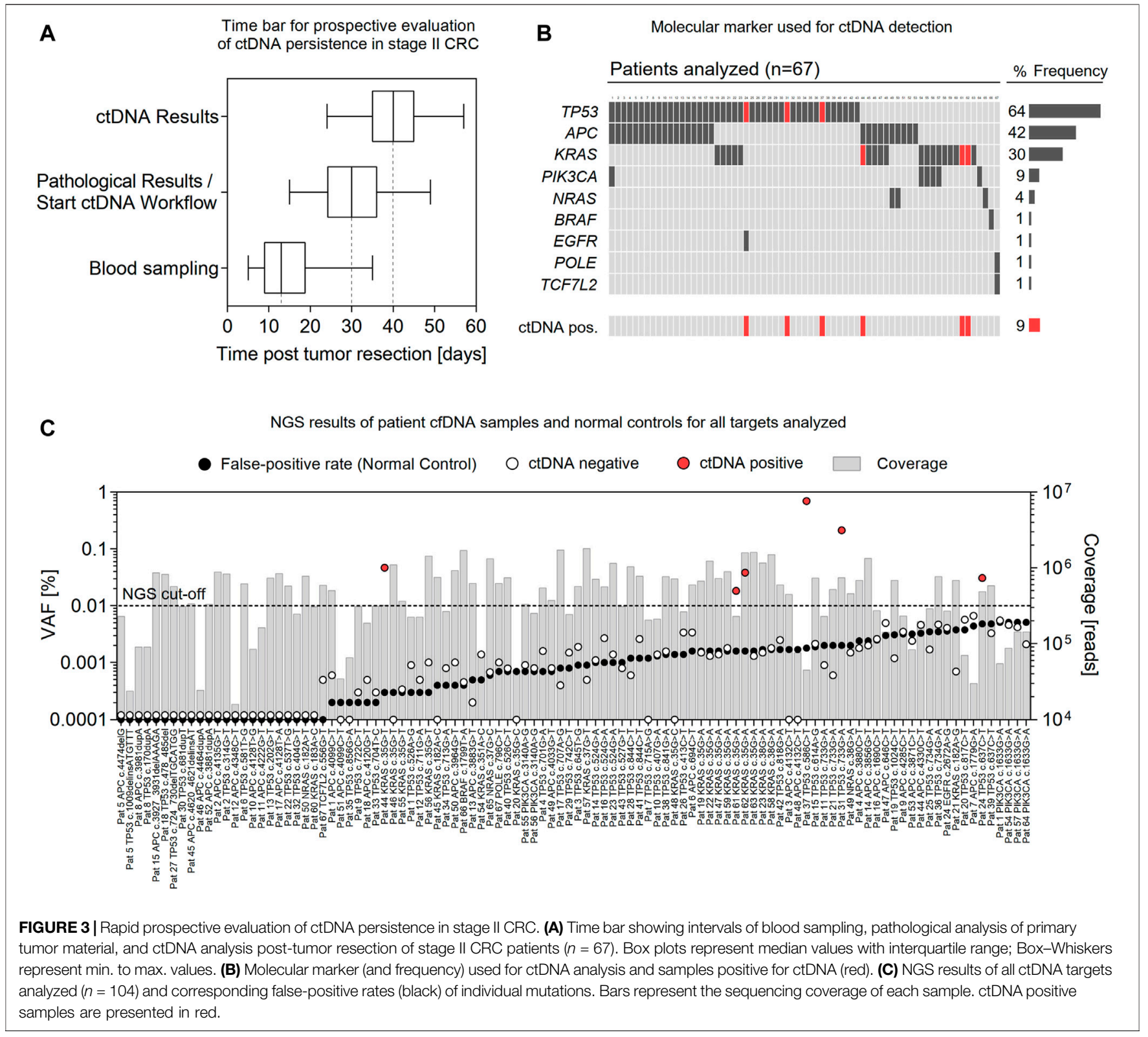

detection of progression. However, for these patients, no plasma samples were available within a period of 5 months prior to clinical relapse in our retrospective analysis, emphasizing the importance of appropriate sampling intervals (i.e., every 2 months) for monitoring. The only patient (Pat. 5) without detectable ctDNA at relapse carried a KRAS hot spot mutation found at subclonal levels in the primary tumor tissue, pointing to the presence of intra-tumoral heterogeneity and clonal selection during cancer progression (Khan et al., 2018). Likewise, a negative selection of RAS mutations due to selective pressure after treatment has been observed in hematological malignancies (Oshima et al., 2016) and in the plasma of CRC patients (Spindler et al., 2014), with implications for RAS testing during adjuvant therapy (Thierry et al., 2017; Normanno et al., 2018). In this regard, the application of whole gene panels for ctDNA monitoring can improve the detection of emerging CRC clones and the assessment of clonal tumor evolution, which might minimize false-negative ctDNA results but increases costs and analysis time to achieve sufficient sensitivity (Malapelle et al., 2017). Moreover, false-negative postoperative ctDNA results might occur in patients with low-shedding tumors, which can be excluded from ctDNA-guided treatment by analyzing presurgery blood samples (Naidoo et al., 2021). In addition to biological tumor features, the sensitivity of most ctDNA assays is limited by the available cfDNA template, which in turn depends on efficient extraction procedures (Barták et al., 2019; van der Leest et al., 2020).

In our study, adequate cfDNA processing routinely allowed the extraction of $\sim 10 \mathrm{ng}$ of cfDNA $\mathrm{mL}^{-1}$ plasma in clinical samples of CRC patients (depending on tumor load), which is 
in the range of concentrations typically measured for cfDNA in human plasma (Fleischhacker and Schmidt, 2007). As we used $2.5 \mathrm{ml}$ plasma for cfDNA extraction, 8,333 alleles per patient sample were available for PCR amplification (assuming $\sim 3 \mathrm{pg}$ of DNA per haploid human genome and $10 \mathrm{ng}$ cfDNA $\mathrm{mL}^{-1}$ plasma), which results in a theoretical limit of $0.012 \%$ VAF for the quantification of heterozygous variants. In line, sensitivity for low-level ctDNA increased by increasing the input of cfDNA as PCR template and by the integration of sequencing reads from triplicate PCRs, pointing to an "all-ornothing" positive amplification in samples with low mutant DNA copy numbers and a potential benefit of binning for barcoded sequencing reads. In combination with sufficient sequencing depth ( $\geq 100.000$ reads), the sensitivity of our error-reduced NGS approach $(0.01 \%$ VAF $)$ is one to two orders of magnitude higher than conventional targeted NGS procedures (typically 0.1-1\% error) (Rachiglio et al., 2016; Osumi et al., 2019) and similar to sensitivities determined for digital droplet PCR (Demuth et al., 2018) or for other modified NGS protocols (Yeh et al., 2020). Turnaround time for analysis (24-48 h) and costs ( $\sim 5 € €$ per sample) of our NGS-based procedure are slightly higher than ddPCR $(8 \mathrm{~h},<20 €)$ and are in the range of other PCR-based approaches with similar sensitivities for cfDNA analysis such as BEAMing (Demuth et al., 2018; Garcia et al., 2018). However, in comparison to other ctDNA assays (Malapelle et al., 2017; Kastrisiou et al., 2019), our targeted NGS procedure allows a versatile (i.e., simple multiplexing) analysis of ctDNA, which is relevant for applied clinical diagnostics.

From the theoretical considerations outlined earlier, a further increase in sensitivity to $0.001 \%$ VAF would require template concentrations of $\sim 300 \mathrm{ng}$ of cfDNA (100.000 haploid copies) for PCR-based methods tracking a single molecular marker, which is likely unrealistic using common volumes of whole blood $(10 \mathrm{ml})$ for cfDNA preparation. Recently, a patient-specific sequencing approach integrating variant reads of multiple mutated genomic loci reported on sensitivities of $0.001-0.0001 \%$ for ctDNA monitoring, which in part depended on tumor mutational burden and high plasma input material (Wan et al., 2020). Accordingly, the parallel analysis of multiple ctDNA markers (i.e., up to 3 markers per patient in our prospective study) offers additional capacity to increase sensitivity for clinical implementation. Based on the methodological improvements and interim results in the present study, a multicenter, prospective, randomized trial (CIRCULATE, NCT04089631, phase III), which started in June 2020, is currently evaluating circulating tumor DNA-based decision for randomized adjuvant treatment in colon cancer stage II (Folprecht et al., 2020).

Taken together, we provide a robust and easily adaptable approach for the sensitive detection of plasma ctDNA in clinical routine, using optimized pre-analytical workup of samples for efficient cfDNA preparation and subsequent error-reduced targeted deep sequencing. Using optimized conditions, the detection of postoperative ctDNA for early prediction of
CRC persistence or recurrence is feasible with excellent sensitivity and specificity at frequencies in the range of $0.01-0.1 \%$ and superior compared to standard imaging-based surveillance.

\section{DATA AVAILABILITY STATEMENT}

The original contributions presented in the study are included in the article/supplementary materials, further inquiries can be directed to the corresponding author.

\section{ETHICS STATEMENT}

The studies involving human participants were reviewed and approved by the Local Ethical Board of the University Hospital Dresden. The patients/participants provided their written informed consent to participate in this study.

\section{AUTHOR CONTRIBUTIONS}

GF and CT contributed to the conception of the work; SM and MM helped with sample collection; SS, SM, CS, CK, DA, AT, AR, and GB acquired and analyzed the data; SS and CS assisted with bioinformatics analysis; SS, SM, GF, CT, MB, and GE helped with interpretation of the data; SS drafted the manuscript; and all authors have seen and approved the submitted manuscript version.

\section{FUNDING}

This study was supported by the BMBF (Grant 031A424) and the National Center for Tumor Diseases (NCT), Heidelberg, Partner Site Dresden, Germany. CIRCULATE is funded by the German Federal Ministry for Education and Research (Bundesministerium für Bildung und Forschung) with grant number 01KG1817.

\section{ACKNOWLEDGMENTS}

For excellent technical assistance, we thank Marita Hartwig, Marika Böhm, and Peggy Grassmel.

\section{SUPPLEMENTARY MATERIAL}

The Supplementary Material for this article can be found online at: https://www.frontiersin.org/articles/10.3389/fgene.2021.811291/ full\#supplementary-material 


\section{REFERENCES}

Barták, B. K., Kalmár, A., Galamb, O., Wichmann, B., Nagy, Z. B., Tulassay, Z., et al. (2019). Blood Collection and Cell-free DNA Isolation Methods Influence the Sensitivity of Liquid Biopsy Analysis for Colorectal Cancer Detection. Pathol. Oncol. Res. 25 (3), 915-923. Epub 2018 Jan 27. PMID: 29374860. doi:10.1007/ s12253-018-0382-z

Bettegowda, C., Sausen, M., Leary, R. J., Kinde, I., Wang, Y., Agrawal, N., et al. (2014). Detection of Circulating Tumor DNA in Early- and Late-Stage Human Malignancies. Sci. Transl. Med. 6 (224), 224ra24. PMID: 24553385; PMCID: PMC4017867. doi:10.1126/scitranslmed.3007094

Böckelman, C., Engelmann, B. E., Kaprio, T., Hansen, T. F., and Glimelius, B. (2015). Risk of Recurrence in Patients with colon Cancer Stage II and III: a Systematic Review and Meta-Analysis of Recent Literature. Acta Oncol. 54 (1), 5-16. Epub 2014 Nov 28. PMID: 25430983. doi:10.3109/ 0284186X.2014.975839

Crowley, E., Di Nicolantonio, F., Loupakis, F., and Bardelli, A. (2013). Liquid Biopsy: Monitoring Cancer-Genetics in the Blood. Nat. Rev. Clin. Oncol. 10 (8), 472-484. Epub 2013 Jul 9. PMID: 23836314. doi:10.1038/nrclinonc.2013.110

Demuth, C., Spindler, K.-L. G., Johansen, J. S., Pallisgaard, N., Nielsen, D., Hogdall, E., et al. (2018). Measuring KRAS Mutations in Circulating Tumor DNA by Droplet Digital PCR and Next-Generation Sequencing. Translational Oncol. 11 (5), 1220-1224. Epub 2018 Aug 4. PMID: 30086420; PMCID: PMC6085225. doi:10.1016/j.tranon.2018.07.013

El Messaoudi, S., Mouliere, F., Du Manoir, S., Bascoul-Mollevi, C., Gillet, B., Nouaille, M., et al. (2016). Circulating DNA as a Strong Multimarker Prognostic Tool for Metastatic Colorectal Cancer Patient Management Care. Clin. Cancer Res. 22 (12), 3067-3077. Epub 2016 Feb 4. PMID: 26847055. doi:10.1158/10780432.CCR-15-0297

Fleischhacker, M., and Schmidt, B. (2007). Circulating Nucleic Acids (CNAs) and Cancer-A Survey. Biochim. Biophys. Acta (Bba) - Rev. Cancer 1775 (1), 181-232. Epub 2006 Oct 7. PMID: 17137717. doi:10.1016/j.bbcan.2006.10.001

Folprecht, G., Reinacher-Schick, A., Tannapfel, A., Weitz, J., Kossler, T., Weiss, L., et al. (2020). Circulating Tumor DNA-Based Decision for Adjuvant Treatment in colon Cancer Stage II Evaluation: (CIRCULATE-Trial) AIO-KRK-0217. Jco 38 (4_Suppl. 1), TPS273. doi:10.1200/jco.2020.38.4_suppl.tps273

Garcia, J., Forestier, J., Dusserre, E., Wozny, A.-S., Geiguer, F., Merle, P., et al. (2018). Cross-platform Comparison for the Detection of RAS Mutations in cfDNA (ddPCR Biorad Detection Assay, BEAMing Assay, and NGS Strategy). Oncotarget 9 (30), 21122-21131. doi:10.18632/oncotarget.24950

Kastrisiou, M., Zarkavelis, G., Pentheroudakis, G., and Magklara, A. (2019). Clinical Application of Next-Generation Sequencing as A Liquid Biopsy Technique in Advanced Colorectal Cancer: A Trick or A Treat? Cancers 11 (10), 1573. PMID: 31623125; PMCID: PMC6826585. doi:10.3390/ cancers11101573

Khan, K. H., Cunningham, D., Werner, B., Vlachogiannis, G., Spiteri, I., Heide, T., et al. (2018). Longitudinal Liquid Biopsy and Mathematical Modeling of Clonal Evolution Forecast Time to Treatment Failure in the PROSPECT-C Phase II Colorectal Cancer Clinical Trial. Cancer Discov. 8 (10), 1270-1285. Epub 2018 Aug 30. PMID: 30166348; PMCID: PMC6380469. doi:10.1158/2159-8290.CD17-0891

Luo, H., Zhao, Q., Wei, W., Zheng, L., Yi, S., Li, G., et al. (2020). Circulating Tumor DNA Methylation Profiles Enable Early Diagnosis, Prognosis Prediction, and Screening for Colorectal Cancer. Sci. Transl. Med. 12 (524), eaax7533. doi:10.1126/scitranslmed.aax7533

Malapelle, U., Mayo de-Las-Casas, C., Rocco, D., Garzon, M., Pisapia, P., JordanaAriza, N., et al. (2017). Development of a Gene Panel for Next-Generation Sequencing of Clinically Relevant Mutations in Cell-free DNA from Cancer Patients. Br. J. Cancer 116 (6), 802-810. doi:10.1038/bjc.2017.8

Medina Diaz, I., Nocon, A., Mehnert, D. H., Fredebohm, J., Diehl, F., and Holtrup, F. (2016). Performance of Streck cfDNA Blood Collection Tubes for Liquid Biopsy Testing. PLoS One 11 (11), e0166354. PMID: 27832189; PMCID: PMC5104415. doi:10.1371/journal.pone.0166354

Naidoo, M., Gibbs, P., and Tie, J. (2021). ctDNA and Adjuvant Therapy for Colorectal Cancer: Time to Re-invent Our Treatment Paradigm. Cancers 13 (2), 346. PMID: 33477814; PMCID: PMC7832902. doi:10.3390/cancers13020346
Normanno, N., Esposito Abate, R., Lambiase, M., Forgione, L., Cardone, C., Iannaccone, A., et al. and CAPRI-GOIM Investigators (2018). RAS Testing of Liquid Biopsy Correlates with the Outcome of Metastatic Colorectal Cancer Patients Treated with First-Line FOLFIRI Plus Cetuximab in the CAPRI-GOIM Trial. Ann. Oncol. 29 (1), 112-118. PMID: 28950295. doi:10.1093/annonc/ $\operatorname{mdx} 417$

Oshima, K., Khiabanian, H., da Silva-Almeida, A. C., Tzoneva, G., Abate, F., Ambesi-Impiombato, A., et al. (2016). Mutational Landscape, Clonal Evolution Patterns, and Role of RAS Mutations in Relapsed Acute Lymphoblastic Leukemia. Proc. Natl. Acad. Sci. USA 113 (40), 11306-11311. Epub 2016 Sep 21. PMID: 27655895; PMCID: PMC5056035. doi:10.1073/ pnas. 1608420113

Osumi, H., Shinozaki, E., Takeda, Y., Wakatsuki, T., Ichimura, T., Saiura, A., et al. (2019). Clinical Relevance of Circulating Tumor DNA Assessed through Deep Sequencing in Patients with Metastatic Colorectal Cancer. Cancer Med. 8 (1), 408-417. Epub 2018 Dec 21. PMID: 30575318; PMCID: PMC6346227. doi:10.1002/cam4.1913

Rachiglio, A. M., Abate, R. E., Sacco, A., Pasquale, R., Fenizia, F., Lambiase, M., et al. (2016). Limits and Potential of Targeted Sequencing Analysis of Liquid Biopsy in Patients with Lung and colon Carcinoma. Oncotarget 7 (41), 66595-66605. PMID: 27448974; PMCID: PMC5341823. doi:10.18632/ oncotarget.10704

Reinert, T., Henriksen, T. V., Christensen, E., Sharma, S., Salari, R., Sethi, H., et al. (2019). Analysis of Plasma Cell-free DNA by Ultradeep Sequencing in Patients with Stages I to III Colorectal Cancer. JAMA Oncol. 5 (8), 1124-1131. Epub ahead of print. doi:10.1001/jamaoncol.2019.0528

Reinert, T., Schøler, L. V., Thomsen, R., Tobiasen, H., Vang, S., Nordentoft, I., et al. (2016). Analysis of Circulating Tumour DNA to Monitor Disease burden Following Colorectal Cancer Surgery. Gut 65 (4), 625-634. doi:10.1136/gutjnl2014-308859

Schøler, L. V., Reinert, T., Ørntoft, M.-B. W., Kassentoft, C. G., Árnadóttir, S. S., Vang, S., et al. (2017). Clinical Implications of Monitoring Circulating Tumor DNA in Patients with Colorectal Cancer. Clin. Cancer Res. 23 (18), 5437-5445. Epub 2017 Jun 9. PMID: 28600478. doi:10.1158/10780432.CCR-17-0510

Schwarzenbach, H., Hoon, D. S. B., and Pantel, K. (2011). Cell-free Nucleic Acids as Biomarkers in Cancer Patients. Nat. Rev. Cancer 11 (6), 426-437. Epub 2011 May 12. PMID: 21562580. doi:10.1038/nrc3066

Siravegna, G., Mussolin, B., Buscarino, M., Corti, G., Cassingena, A., Crisafulli, G., et al. (2015). Erratum: Clonal Evolution and Resistance to EGFR Blockade in the Blood of Colorectal Cancer Patients. Nat. Med. 21 (7), 827. Erratum for: Nat Med. 2015 Jul;21(7):795-827. PMID: 26151329. doi:10.1038/nm0715$827 \mathrm{~b}$

Spindler, K.-L. G., Pallisgaard, N., Andersen, R. F., and Jakobsen, A. (2014). Changes in Mutational Status during Third-Line Treatment for Metastatic Colorectal Cancer-Results of Consecutive Measurement of Cell Free DNA,KRASandBRAFin the Plasma. Int. J. Cancer 135 (9), 2215-2222. Epub 2014 Apr 17. PMID: 24659028. doi:10.1002/ijc.28863

Stasik, S., Schuster, C., Ortlepp, C., Platzbecker, U., Bornhäuser, M., Schetelig, J., et al. (2018). An Optimized Targeted Next-Generation Sequencing Approach for Sensitive Detection of Single Nucleotide Variants. Biomol. Detect. Quantification 15, 6-12. PMID: 29349042; PMCID: PMC5766748. doi:10.1016/j.bdq.2017.12.001

Strickler, J. H., Loree, J. M., Ahronian, L. G., Parikh, A. R., Niedzwiecki, D., Pereira, A. A. L., et al. (2018). Genomic Landscape of Cell-free DNA in Patients with Colorectal Cancer. Cancer Discov. 8 (2), 164-173. doi:10.1158/2159-8290.CD17-1009

Thierry, A. R., El Messaoudi, S., Mollevi, C., Raoul, J. L., Guimbaud, R., Pezet, D., et al. (2017). Clinical Utility of Circulating DNA Analysis for Rapid Detection of Actionable Mutations to Select Metastatic Colorectal Patients for Anti-EGFR Treatment. Ann. Oncol. 28 (9), 2149-2159. PMID: 28911069. doi:10.1093/ annonc/mdx330

Tie, J., Cohen, J. D., Wang, Y., Christie, M., Simons, K., Lee, M., et al. (2019). Circulating Tumor DNA Analyses as Markers of Recurrence Risk and Benefit of Adjuvant Therapy for Stage III Colon Cancer. JAMA Oncol. 5 (12), 1710-1717. PMID: 31621801; PMCID: PMC6802034. doi:10.1001/ jamaoncol.2019.3616 
Tie, J., Kinde, I., Wang, Y., Wong, H. L., Roebert, J., Christie, M., et al. (2015). Circulating Tumor DNA as an Early Marker of Therapeutic Response in Patients with Metastatic Colorectal Cancer. Ann. Oncol. 26 (8), 1715-1722. Epub 2015 Apr 7. PMID: 25851626; PMCID: PMC4511218. doi:10.1093/ annonc/mdv177

Tie, J., Wang, Y., Tomasetti, C., Li, L., Springer, S., Kinde, I., et al. (2016). Circulating Tumor DNA Analysis Detects Minimal Residual Disease and Predicts Recurrence in Patients with Stage II colon Cancer. Sci. Transl. Med. 8 (346), 346ra92. PMID: 27384348; PMCID: PMC5346159. doi:10.1126/ scitranslmed.aaf6219

van der Leest, P., Boonstra, P. A., ter Elst, A., van Kempen, L. C., Tibbesma, M., Koopmans, J., et al. (2020). Comparison of Circulating Cell-free DNA Extraction Methods for Downstream Analysis in Cancer Patients. Cancers 12 (5), 1222. PMID: 32414097; PMCID: PMC7281769. doi:10.3390/cancers12051222

Wan, J. C. M., Heider, K., Gale, D., Murphy, S., Fisher, E., Mouliere, F., et al. (2020). ctDNA Monitoring Using Patient-specific Sequencing and Integration of Variant Reads. Sci. Transl. Med. 12 (548), eaaz8084. doi:10.1126/ scitranslmed.aaz8084

Wan, J. C. M., Massie, C., Garcia-Corbacho, J., Mouliere, F., Brenton, J. D., Caldas, C., et al. (2017). Liquid Biopsies Come of Age: towards Implementation of Circulating Tumour DNA. Nat. Rev. Cancer 17 (4), 223-238. Epub 2017 Feb 24. PMID: 28233803. doi:10.1038/nrc.2017.7

Wang, Y., Li, L., Cohen, J. D., Kinde, I., Ptak, J., Popoli, M., et al. (2019). Prognostic Potential of Circulating Tumor DNA Measurement in Postoperative Surveillance of Nonmetastatic Colorectal Cancer. JAMA Oncol. 5 (8), 1118-1123. Epub ahead of print. PMID: 31070668; PMCID: PMC6512291. doi:10.1001/jamaoncol.2019.0512
Yeh, Y.-M., Lin, P.-C., Lee, C.-T., Chen, S.-H., Lin, B.-W., Lin, S.-C., et al. (2020) Treatment Monitoring of Colorectal Cancer by Integrated Analysis of Plasma Concentration and Sequencing of Circulating Tumor DNA. Mol. Cancer 19 (1), 150. PMID: 33106181; PMCID: PMC7586655. doi:10.1186/s12943-02001273-8

Conflict of Interest: CT is CEO and co-owner of AgenDix GmbH, a company performing molecular diagnostics. $\mathrm{CS}$ and $\mathrm{CK}$ are employed at AgenDix GmbH.

The remaining authors declare that the research was conducted in the absence of any commercial or financial relationships that could be construed as a potential conflict of interest.

Publisher's Note: All claims expressed in this article are solely those of the authors and do not necessarily represent those of their affiliated organizations, or those of the publisher, the editors, and the reviewers. Any product that may be evaluated in this article, or claim that may be made by its manufacturer, is not guaranteed or endorsed by the publisher.

Copyright (C) 2022 Stasik, Mende, Schuster, Mahler, Aust, Tannapfel, ReinacherSchick, Baretton, Krippendorf, Bornhäuser, Ehninger, Folprecht and Thiede. This is an open-access article distributed under the terms of the Creative Commons Attribution License (CC BY). The use, distribution or reproduction in other forums is permitted, provided the original author(s) and the copyright owner(s) are credited and that the original publication in this journal is cited, in accordance with accepted academic practice. No use, distribution or reproduction is permitted which does not comply with these terms. 